\title{
Mathematical modelling of burning surface in parallel flow of oxidant
}

\author{
Veronika Tyurenkova ${ }^{1,2, * *}$, Nickolay Smirnov ${ }^{1,2}$, and Mariya Smirnova ${ }^{1,2}$ \\ ${ }^{1}$ Federal Science Center Scientific Research Institute for System Studies of Russian Academy of \\ Sciences, 117218 Moscow, Russia \\ ${ }^{2}$ Moscow M.V. Lomonosov State University, 119991, Moscow, Russia
}

\begin{abstract}
The paper presents the problem of condensed material surface burning in a flow of oxidant within the framework of the assumptions of the laminar boundary-layer theory. It is used assumption of fuel gasification and gas phase chemical reacting in a diffusion flame. The regression rate of the material surface in the turbulent and laminar flow regimes is studied. The zones correspond to kinetic and diffusion regime is defined.
\end{abstract}

\section{Introduction}

The condensed fuel burning in an oxidant gas flow occurs in investigations of various processes, for example: burning of fuel in hybrid engines; interaction of hypersonic flying vehicles with the atmosphere; exo- and endothermic reactions in chemical engineering; spreading of flame over the fuel surface, the origination of explosions and the propagation of detonation in unmixed two-phase systems of the gas-film type.

The present work investigates the burning of the flat surface of fuel in an oxidant flow in the absence of an axial pressure gradient. The problem is solved within the framework of the assumptions of the laminar boundary-layer theory. Near the fuel surface in the gas, the boundary layer develops in the interior of which there proceed chemical reactions between the fuel vapours and the oxidant. Since these are exothermic reactions, energy is given up by the boundary layer in the gas. The heat from the heated gas transfers to the fuel layer which, having evaporated, gains access, as a result of diffusion, the boundary layer, where it enters into reaction with the oxidant. As a result of continuous inflow of heat from the gas phase, the fuel becomes warmed up and a temperature boundary layer develops near the phase interface.

\section{Mathematical problem statement}

The main aspect of the problem statement is the model for gasification of solid fuel surface. As for now, there exist two different approaches, which represent the two limiting case: quasi-equilibrium gasification and absolutely non-equilibrium gasification.

Let the gasification process be described by Hertz-Knudsen formula

\footnotetext{
* Corresponding author: tyurenkova.v.v@yandex.ru
} 


$$
\dot{m}_{N}=k\left(p_{N}^{*}-p_{N}\right), k=\delta \sqrt{\frac{m_{N}}{2 \pi R T_{w}}}=\delta_{o} \sqrt{\frac{m_{N}}{2 \pi R T_{w}}} e^{-\frac{E_{\delta}}{R T_{w}}},
$$

where $p_{N}$ is partial pressure of fuel vapor near the phase interface, $T_{w}$ - temperature of the interface, $p_{N}^{*}$ - phase equilibrium pressure for $T=T_{w}, m_{N}$ - molar mass of fuel, $R$ universal gas constant, $\delta$ - accommodation coefficient, $\delta_{0}$ - maximal allowable value for accommodation coefficient, $E_{\delta}$ - activation energy for gasification. The limiting cases of for formula (1) are the following:

(1) For relatively high partial pressure of the fuel vapor in the flow an equilibrium state at the interface can be achieved $\left(p_{N}^{*}\left(T_{w}\right)=p_{N}\right)$ under which the numbers of gasifying and condensing molecules are equal. However, in the presence of heat flux phase transition rate can be determined then based on the energy balance, which is not taken into account by classical Hertz-Knudsen model.

(2) For relatively low partial pressure of fuel vapor $\left(p_{N}<<p_{N}^{*}\left(T_{w}\right)\right)$ formula (1) can be presented in the form

$$
\dot{m}_{N}=\delta_{o} \sqrt{\frac{m_{N}}{2 \pi R T_{w}}} p_{N}^{*} e^{-\frac{E_{\delta}}{R T_{w}}}
$$

which is the gasification (or pyrolysis) formula accepted for many solid fuels.

(3) For the intermediate case the partial pressure being non-zero, and, yet, rather far from its equilibrium value, the formula (1) should be used along with the energy flux conservation condition to determine the unknown values of $\dot{m}_{N}$ and $T_{w}$. This generalized non-equilibrium model was developed and applied for droplets combustion in $[1,2]$.

The solution of the problem will be based on the concept of diffusion flame and will use analytical research methodology developed in [1-2]. Contrary to symmetric problems solved in [1-2] the present problem statement will differ greatly by incorporating the flow dynamics in the frame of boundary layer approximation, and accounting for fuel heating dynamics on moving from the flame tip.

Let the rectangular coordinate systems $(\mathrm{x}, \mathrm{y})$ and $(\tilde{x}, \tilde{y})$ be chosen so that the axis $\mathrm{x}$ would be coincident with the phase interface and would be aligned with the oxidant flow, while the axis $y$ and $\tilde{y}$ in the gas and liquid, respectively, would be aligned against the interface. The coordinate origin coincides with the start of the boundary layer.

Then, the equations of a boundary layer in a gas, provided that the Prandtl and Schmidt numbers are equal to unity, are:

$$
\begin{gathered}
\frac{\partial \rho u}{\partial x}+\frac{\partial \rho v}{\partial y}=0, \rho u \frac{\partial u}{\partial x}+\rho v \frac{\partial u}{\partial y}=\frac{\partial}{\partial y} \mu \frac{\partial u}{\partial y} \\
\rho u \frac{\partial Y_{i}}{\partial x}+\rho v \frac{\partial Y_{i}}{\partial y}=\frac{\partial}{\partial y} \mu \frac{\partial Y_{i}}{\partial y}+\omega_{i} ; i=1, \ldots, N, \rho u \frac{\partial H}{\partial x}+\rho v \frac{\partial H}{\partial y}=\frac{\partial}{\partial y} \mu \frac{\partial H}{\partial y} p=\rho R T \sum_{i=1}^{N} Y_{i} / m_{i}
\end{gathered}
$$

where $H=\sum_{i=1}^{N} Y_{i}\left(c_{p i} T+h_{i}^{o}\right)+\frac{u^{2}}{2}$ is the total specific enthalpy of the mixture and, a $h_{i}=c_{p i} T+h_{i}^{o}$ is the $\mathrm{i}$-th component specific enthalpy. In the case of solid fuel the equations for a boundary layer at constant heat capacity $c_{f}$, viscosity $\mu_{f}$ and thermal conductivity $\lambda_{f}$ reduce to energy equation: 


$$
\tilde{v}_{f} \frac{\partial \tilde{T}}{\partial \tilde{y}}=\frac{\lambda}{\rho c} \frac{\partial^{2} \tilde{T}}{\partial \tilde{y}^{2}}
$$

where $v_{f}=v_{f}(x)$ is the velocity of vertical movement of fuel, deriving from evaporation, because coordinate system in the vertical direction is moving with the phase interface.

The conditions on the outer edge of a boundary layer in a gas $(y \rightarrow+\infty)$ : $T=T_{e}, p=p_{e}, u=u_{e}, Y_{1}=Y_{1 e}, Y_{1 e}+Y_{2 e}=1$. The conditions on the edge of a boundary layer in a fuel $(\tilde{y} \rightarrow+\infty): \tilde{T}=T_{\infty}, \tilde{u}=u_{\infty}=0$. The balance relationships at the phase interface ( $y=0 ; x>0)$ are following: total mass flux conservation condition $(\rho v)_{w}=-(\rho \tilde{v})_{f w}$, noslip condition $u=\tilde{u}=0$, condition of tangential stresses continuity $\tau=-\tau_{f w},\left(\tau=-\mu \frac{\partial Y_{i}}{\partial y}\right)$.

Condition of variation in the mass flow of each of the components in the absence of heterogeneous reactions $(\rho v)_{w}\left(Y_{i w}-Y_{i w f}\right)-(\rho D)_{w}\left(\frac{\partial Y_{i}}{\partial y}\right)_{w}=0$. Total energy flux conservation condition under assumptions that: the fuel is one-component $\left(Y_{N f w}=1\right)$, the heterogeneous reactions are absent $\left(g_{i w}=0\right)$, the squares of the velocity components on the axis $y \quad\left(v_{f w}^{2}, v_{w}^{2}\right)$ may be neglected, has the following form $\lambda_{f}\left(\frac{\partial \tilde{T}}{\partial \tilde{y}}\right)_{f w}=-\lambda\left(\frac{\partial T}{\partial y}\right)_{w}+(\rho v)_{w} h_{L}$.

Define the interface temperature $T_{w}$ as the temperature of equilibrium evaporation of fuel at a pressure $p_{N w}$, which is equal to the partial vapor pressure of the vaporized fuel at the interface $p_{N_{w}}=Y_{N_{w}} p_{e} \frac{m_{w}}{m_{N}}$. The molecular weight of the mixture $m_{w}$ is determined by the following equation $\frac{1}{m_{w}}=\sum_{i=1}^{N} \frac{Y_{i w}}{m_{i}}$. The pressure $p_{N w}$ could be defined from the ClausiusClapeyron equation $\frac{p_{e}}{p_{N W}}=\left(\frac{T_{k e}}{T_{w}}\right)^{\Delta c_{p} m_{N} / R} \exp \left[\frac{\Delta c_{p} m_{N} T_{k p}}{R}\left(\frac{1}{T_{k e}}-\frac{1}{T_{w}}\right)\right]$, where $T_{k e}$ - is the phase transition temperature at the pressure $p_{e}, T_{k p}$ - critical temperature.

\section{Self-similar problem solution}

Introduce new independent variables $z=\int_{o}^{y} \rho d y, \varsigma=\rho \mu x$, the dependent variables $w=\frac{1}{\rho \mu}\left(\rho v+u \frac{\partial z}{\partial x}\right)$ and steam functions $\Psi$ so that $u=\frac{\partial \Psi}{\partial z} ; w=-\frac{\partial \Psi}{\partial \varsigma}$.

Then, the first equations of system (3) are satisfied identically, while the remaining equations, after the transition to the similarity variables $\eta=z \sqrt{\frac{u_{e}}{2 \varsigma}} ; \Psi=\sqrt{2 \varsigma u_{e}} g(\eta)$ are reduced to the ordinary differential equations

$$
g^{\prime \prime \prime}(\eta)+g^{\prime \prime}(\eta) g(\eta)=0
$$


with boundary conditions

$$
g^{\prime}(0)=0, g^{\prime}(\infty)=1, g(0)=-B g^{\prime \prime}(0),
$$

where $B=\frac{(\rho v)_{w} u_{e}}{\tau_{w}}$ is the dimensionless parameter of mass transfer.

The equation (4) can be integrated: $-\left(\lambda \frac{\partial \tilde{T}}{\partial \tilde{y}}\right)_{f W}=(\rho \tilde{v})_{f} c_{f}\left(T_{\infty}-T_{W}\right)=(\rho v)_{w} c_{f}\left(T_{w}-T_{\infty}\right)$.

Thus the equation (5) and boundary conditions (6) make it possible to solve the problem. The surface temperature $T_{w}$ and the mass transfer parameter B are determined by the equations $B=Y_{1 e} \Phi_{N}+\sum_{i=2}^{N-1} \frac{m_{N}}{m_{i}}\left(Y_{i e}-Y_{1 e} \Phi_{i}\right)\left(\frac{p_{e}}{p_{N W}}-1\right)^{-1}$ and $B\left[c_{f}\left(T_{w}-T_{\infty}\right)+h_{L}\right]=\hat{c}_{p e}\left(T_{e}-T_{w}\right)+\frac{u_{e}^{2}}{2}+Y_{1 e}\left(\Phi_{N} \Delta H+\Delta C_{p} T_{w}\right)$.

\section{Regression rate}

The regression rate is an important parameter for the hybrid rocket motor, and many efforts have been focused to study the regression rate. The required rate can be determined by the Arrhenius law $\dot{r}=A \exp \left(-E_{a} / R T_{w}\right)$, where $A, E_{a}, R$ are constants, $T_{w}$ is the temperature at the interface, as it is done in many papers. Considering the results derived in the previous chapter, we obtain an analytical solution for regression rate other than described. The regression rate can be determined by the Arrhenius law $\dot{r}=A \exp \left(-E_{a} / R T_{w}\right)$, where the parameters $A, E_{a}$ for HTPB is $A=0.01104, E_{a}=20523.8$ for $T_{w}>722 K$ and $A=3.96480, E_{a}=55803$ for $T_{w}<722 K$.

Fig. 1 shows the regression rate profiles, which were obtained, first, based on self-similar

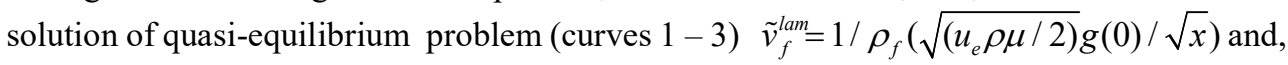
second, based strictly non-equilibrium model (Arrhenius law, curve 4). It is seen from Fig. 1 , that the regression rate, determined by the Arrhenius law, is constant, which is valid for very high diffusion fluxes, when kinetics of gasification becomes the limiting factor. In burning solid fuel surface kinetic regime is realized not for the whole specimen, but only near the tip of boundary layer. At longer distances from the tip diffusion fluxes decrease thus becoming the limiting factor. Since kinetic regime (2) presents the fastest gasification rate, the self-similar solution near the tip surpassing the kinetic regression rate cannot be valid, as well as kinetic regime is not valid at longer distances from the tip, where it is limited by diffusion fluxes.

Depending on the flow velocity the flow may be laminar and turbulent in the boundary layer. As a rule, there is a combined flow in the boundary layer characterized by transition from laminar to turbulent regimes. The position of the transition zone can be approximately determined by the critical value of the local Reynolds number $\operatorname{Re}=\rho u_{e} x / \mu$. To find an analytical solution for the regression rate at a turbulent flow mode, we determine the tangential stress in the absence of mass transfer parameter $(\mathrm{B}=0) \tau_{w o}=0.0296 \cdot \rho u_{e}^{2} \cdot \mathrm{Re}^{-1 / 5}$. A link between tangential stresses in the presence of mass transfer $\tau_{w}$ and in the absence of one $\tau_{w o}$ can be obtained for small values of mass transfer parameter $\mathrm{B} \tau_{w} / \tau_{w o}=\ln (1+B) / B$ Then the regression rate for turbulence flow regime
$(\rho \tilde{v})_{f}=\left(\tau_{w} B\right) / u_{e}=0.0296 \cdot \rho u_{e} \ln (1+B) \cdot \mathrm{Re}^{-1 / 5}$. Fig. 2 shows the comparison of the 
analytical solution with numerical solutions for regression rate of HTPB fuel. The numerical calculations are based on two methods of modeling the mass regression rate. In this problem, numerical results demonstrate a good coincidence with exact analytical solutions as well as with experimental data.

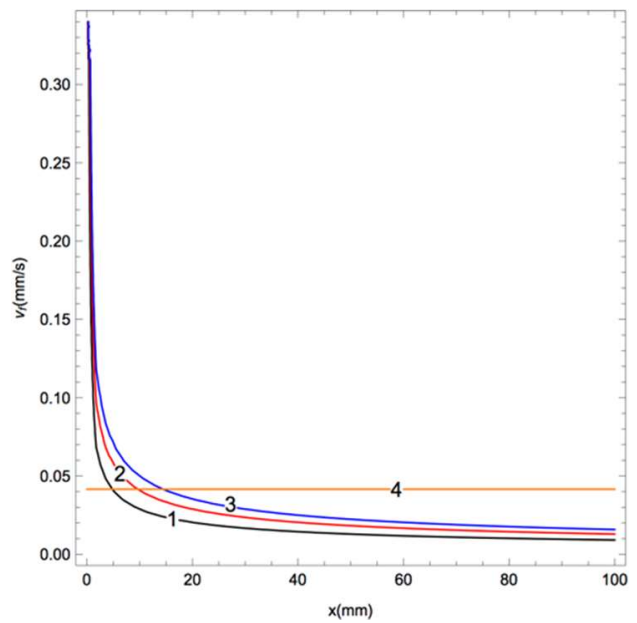

Fig.1. The profiles of regression rate, built on the basis of analytical formula (curve 1-3) and the Arrhenius law (curve 4) (curve $1-u_{e}=5 \mathrm{~m} / \mathrm{s}$, curve $2-u_{e}=10 \mathrm{~m} / \mathrm{s}$, curve $3-u_{e}=15 \mathrm{~m} / \mathrm{s}$ ).

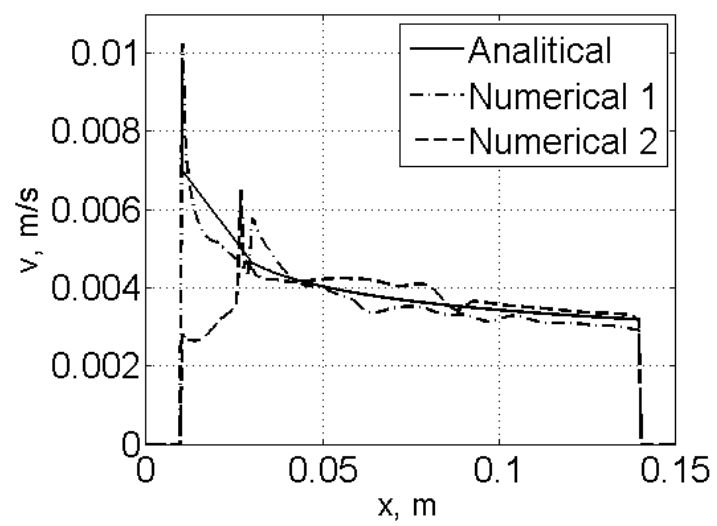

Fig.2. Analytical and numerical solutions for regression rate of HTPB fuel $\left(u_{e}=1067 \mathrm{~m} / \mathrm{s}\right)$.

The authors gratefully acknowledge the financial support from the Russian Foundation for Basic Research (Grant № 17-07-00549).

\section{References}

1. V.V.Tyurenkova. Acta Astronaut. 75, 78-84, (2012).

2. V.V.Tyurenkova, N.N. Smirnov, V.M. Guendugov. Acta Astronaut. 83, 208-215, (2013). 